\title{
DETERMINAÇÃO SIMULTÂNEA DE CÁTIONS EMPREGANDO ELETROFORESE CAPILAR COM DETECÇÃO CONDUTOMÉTRICA SEM CONTATO EM EQUIPAMENTO CONSTRUÍDO EM LABORATÓRIO
}

\author{
Leandro M. de Carvalho*, Cristiane Spengler, Ana Paula S. de Lima, Alice Raabe, Mariele Martini, Paulo C. do \\ Nascimento e Denise Bohrer \\ Departamento de Química, Universidade Federal de Santa Maria, CP 5051, 97110-970 Santa Maria - RS, Brasil
}

Recebido em 2/10/08; aceito em 3/4/09; publicado na web em 30/9/09

\begin{abstract}
SIMULTANEOUS DETERMINATION OF CATIONS BY CAPILLARY ELECTROPHORESIS WITH CONTACTLESS CONDUCTIVITY DETECTION PERFORMED IN A HOME-MADE EQUIPMENT. This work describes the development of a home-made capillary electrophoresis (CE) system based on the capacitively coupled contactless conductivity detection $\left(\mathrm{C}^{4} \mathrm{D}\right)$ for the separation of the metallic species $\mathrm{Zn}^{2+}, \mathrm{Cr}^{3+}, \mathrm{Pb}^{2+}, \mathrm{Cd}^{2+}, \mathrm{Co}^{2+}, \mathrm{Cu}^{2+}, \mathrm{Ni}^{2+} \mathrm{e} \mathrm{Tl}^{+}$. A background electrolyte composed of MES/Histidine $0,02 \mathrm{~mol} \mathrm{~L}^{-1}$ ( $\mathrm{pH}$ 5.0) was optimized for the separation of the metallic species by using organic solvents and complexing agents as additives. The system allowed the determination of the metallic species using MES/Histidine 0,02 $\mathrm{mol} \mathrm{L}^{-1}$ and methanol 5\% (pH 5.0) as a background electrolyte, $15 \mathrm{kV}$ separation voltage and hydrodynamic injection by gravity.
\end{abstract}

Keywords: capillary electrophoresis; contactless conductivity detection; cations.

\section{INTRODUÇÃO}

A eletroforese capilar (CE) tem sido muito empregada como método analítico para a separação de cátions metálicos em diversos tipos de amostras. ${ }^{1} \mathrm{O}$ princípio de separação da CE baseia-se na migração diferenciada de compostos neutros, iônicos ou ionizáveis, mediante a aplicação de um campo elétrico em uma coluna capilar contendo em seu interior uma solução de eletrólitos. A CE é um método de separação atraente na análise de misturas complexas devido às suas características de alta eficiência de separação, baixo consumo de reagentes e amostras, tempos de análise reduzidos, simplicidade do equipamento utilizado e alto grau de automação. ${ }^{2} \mathrm{O}$ trabalho pioneiro em CE para a análise de cátions metálicos foi publicado por Hjertén e colaboradores em 1967, descrevendo a separação de $\mathrm{Bi}^{3+}$ e $\mathrm{Cu}^{2+}$ utilizando ácido lático como agente complexante no eletrólito de trabalho. ${ }^{3}$ A partir de 1983, houve um crescimento expressivo no número de artigos publicados envolvendo aspectos instrumentais e aplicações da CE, principalmente devido ao desenvolvimento rápido da instrumentação e, em parte, ao lançamento do primeiro equipamento comercial completo para CE em $1987 .{ }^{4}$

Nos últimos anos, a detecção condutométrica sem contato, ou detecção oscilométrica, tem recebido uma atenção especial como alternativa à detecção condutométrica convencional. Neste tipo de detecção, um sinal alternado de alta frequência, da ordem de centenas de $\mathrm{kHz}$ a dezenas de $\mathrm{MHz}$, é aplicado entre os dois eletrodos na célula de detecção, medindo-se a corrente elétrica resultante que é função da condutividade da solução. Os eletrodos da célula condutométrica constituem um capacitor, enquanto a solução atua como uma resistência cujo valor depende da sua condutividade elétrica. Como a reatância capacitiva diminui com o aumento da frequência, a impedância do capilar é reduzida, permitindo que as variações de condutividade da solução no interior do capilar sejam observadas mesmo com os eletrodos posicionados do lado externo do capilar. A CE com detecção por condutividade sem contato possibilita a determinação rápida e simultânea de íons metálicos com limites de detecção da ordem de $\mu \mathrm{mol}$ $\mathrm{L}^{-1} \cdot{ }^{5-7}$ Além disso, ela é vantajosa em comparação a outros métodos analíticos de separação, principalmente em relação aos baixos custos envolvidos na construção e manutenção do equipamento.

\footnotetext{
*e-mail: leandrocarvalho@pq.cnpq.br
}

A detecção condutométrica sem contato capacitivamente acoplada $\left(\mathrm{C}^{4} \mathrm{D}\right.$, capacitively coupled contactless conductivity detection), consistindo em um arranjo radial, foi aplicada às técnicas de eletromigração no início dos anos 1980 para a determinação isotacoforética de íons pequenos. ${ }^{8}$ Em 1998, Zemann e colaboradores ${ }^{9}$ e da Silva e do Lago $^{5}$ propuseram, de forma independente, a utilização da $\mathrm{C}^{4} \mathrm{D}$ como método de detecção para CE. Desde então, vários outros autores têm explorado este tipo de sistema de detecção na CE. Nesta configuração, a célula de $\mathrm{C}^{4} \mathrm{D}$ em arranjo axial é baseada em dois eletrodos tubulares, que circundam o capilar de separação e são posicionados lado a lado ao longo do eixo do capilar. Um espaço entre os eletrodos ( $\mathrm{gap}$ ) de poucos milímetros de comprimento ${ }^{5,9}(2$ e $1 \mathrm{~mm}$ ) define o volume de detecção. Como cada eletrodo forma um capacitor com a solução do eletrólito dentro do capilar, é possível passar uma voltagem de corrente alternada de alta frequência através da célula.

Os sistemas CE-C ${ }^{4} \mathrm{D}$ desenvolvidos por da Silva e do $\mathrm{Lago}^{5}$ foram totalmente construídos em laboratório, sendo os primeiros sistemas CE- $\mathrm{C}^{4} \mathrm{D}$ desenvolvidos no Brasil. Desde então, a construção de sistemas $\mathrm{CE}-\mathrm{C}^{4} \mathrm{D}$ tem se tornado uma alternativa muito interessante para laboratórios de pesquisa que estejam interessados no desenvolvimento da instrumentação e de métodos analíticos para CE. Seguindo esta tendência, Böckel e colaboradores ${ }^{7}$ desenvolveram, em 2005, um outro sistema CE-C ${ }^{4}$ D com algumas modificações no detector proposto por da Silva e do Lago $^{5}$ e com aquisição de dados utilizando um potenciostato comercial.

A separação de metais alcalinos, alcalino-terrosos e de transição por $\mathrm{CZE}-\mathrm{C}^{4} \mathrm{D}$ já foi relatada na literatura. ${ }^{5,79-12} \mathrm{Na}$ grande maioria destes trabalhos, soluções tampão contendo uma mistura de ácido 2-N-morfolinoetanossulfônico (MES) e histidina (His) em meio ácido são empregados como eletrólitos de trabalho, que mantêm a condutividade de fundo em níveis relativamente baixos. Geralmente, são adicionados ao eletrólito de trabalho agentes complexantes como éter coroa, ácido $\alpha$-hidroxi-isobutírico (HIBA), ácido lático (Hlac), ácido málico e ácido tartárico para alterar as mobilidades eletroforéticas dos íons metálicos, ${ }^{1,4,6,12-18}$ além de solventes orgânicos como metanol para melhorar a resolução dos sinais no eletroferograma. ${ }^{1,4,5,9,14}$

Este trabalho descreve a construção de um sistema $\mathrm{CE}-\mathrm{C}^{4} \mathrm{D}$ com manipulação de soluções completamente automatizada empregando dois carrosséis de amostras acoplados ao sistema. A detecção por $C^{4} \mathrm{D}$ 
é baseada nos protótipos descritos na literatura. ${ }^{5,7} \mathrm{O}$ sistema desenvolvido foi empregado na análise simultânea dos cátions metálicos $\mathrm{Zn}^{2+}$, $\mathrm{Cr}^{3+}, \mathrm{Pb}^{2+}, \mathrm{Cd}^{2+}, \mathrm{Co}^{2+}, \mathrm{Cu}^{2+}, \mathrm{Ni}^{2+} \mathrm{e} \mathrm{Tl}^{+}$em amostras de água.

\section{PARTE EXPERIMENTAL}

\section{Instrumentação}

O equipamento de eletroforese capilar construído e utilizado neste trabalho foi configurado com os seguintes equipamentos e componentes: uma fonte de alta tensão bipolar (0-30 kV) modelo ARM-30 da marca Bertan (Spellman, New York, USA); um gerador de funções de alta frequência da marca Minipa (modelo MFG-4202); um controlador de temperatura da marca Novus N110 com sensor de temperatura modelo Pt100; um sistema de injeção composto de um carro de impressão de uma impressora Epson Stylus Color 600, uma garra manipuladora LG-KT (Little Grip Kit - Labsis) com servomotor (Hitech HS-422) para o acionamento do carro de impressora e um driver de motor de passos modelo DMP-02; uma bomba de vácuo da marca Prismatec; uma resistência de aparelho secador de cabelo com um ventilador modelo DC $12 \mathrm{~V}$ para circulação de ar quente no interior do equipamento; dois ventiladores modelo DC $12 \mathrm{~V}$ e da marca Golden Cabo para circulação de ar no interior do equipamento; dois carrosséis trocadores de amostras contendo 9 reservatórios e um braço eletromecânico; 3 fontes de alimentação: uma fonte de tensão $24 \mathrm{Vdc}$ (modelo LRI S-10024 - Entrada 115 VAC 1,9A/230 VAC 0,8A 50/60 Hz - Saída +24 $\mathrm{V} 4,5 \mathrm{~A}$ ), uma fonte simétrica de $\pm 12 \mathrm{Vdc}$ para alimentação do detector condutométrico (1 A por saída - Entrada 110 V) e uma fonte de $12 \mathrm{Vdc}$ para alimentação do sistema de injeção (Entrada 110 VAC e Saída 12 Vdc 1 A); um detector de condutividade sem contato $\left(\mathrm{C}^{4} \mathrm{D}\right)$ desenvolvido em laboratório; um capilar de sílica fundida de $75 \mu \mathrm{m}$ de diâmetro interno e $360 \mu \mathrm{m}$ de diâmetro externo (Microtube, São Paulo/SP, Brasil); dois eletrodos de platina de $1 \mathrm{~mm}$ de diâmetro conectados aos terminais de alta tensão e terra da fonte; dois reservatórios (vial) para soluções de $1 \mathrm{~mL}$ de capacidade cada, onde são imersos as extremidades do capilar e os eletrodos de platina; uma placa multifuncional modelo PCI-1711 (Advantech, DA\&C Driver version 2.3) para interfaceamento do equipamento com o software do computador. A Figura 1 mostra de maneira esquemática a configuração do sistema eletroforético construído em laboratório.

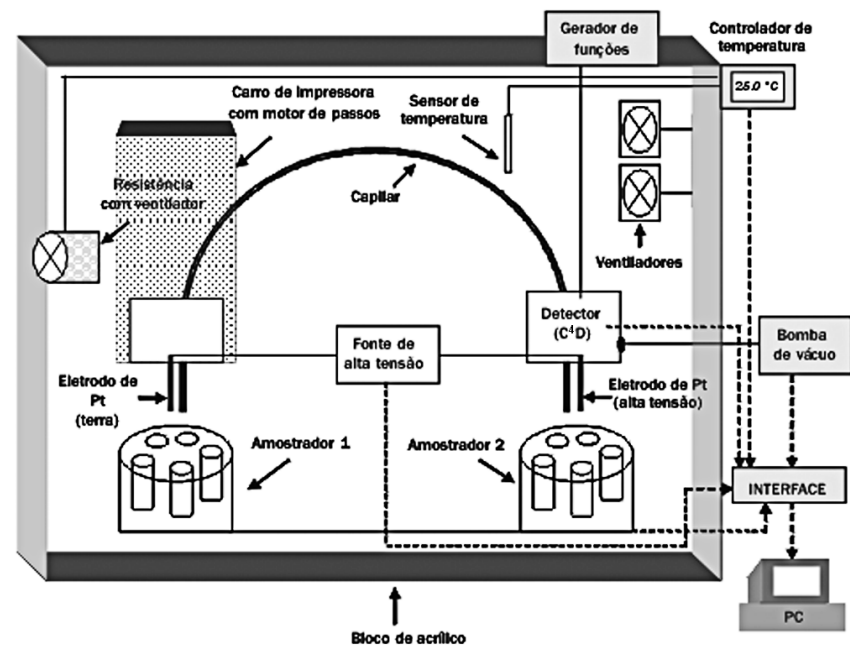

Figura 1. Representação em diagrama de blocos do equipamento de eletroforese capilar construído em laboratório

\section{Descrição do equipamento}

O equipamento foi montado em um bloco de acrílico com dimensões de 70 × 80 × $50 \mathrm{~cm}$. O bloco de acrílico (transparente) possibilita a termostatização do ambiente interno do sistema eletroforético e o trabalho seguro do analista. A fonte de alta tensão aplica uma diferença de potencial (de até $\pm 30 \mathrm{kV}$ ) entre o cátodo e o ânodo (platina), ambos mergulhados na solução do eletrólito de trabalho juntamente com as extremidades do capilar. O comprimento total do capilar foi de 68 $\mathrm{cm}$, sendo a distância entre o ponto de injeção e o detector de $46 \mathrm{~cm}$. Com relação à operação básica do equipamento, a Figura 2 mostra as duas extremidades do equipamento contendo 2 peças projetadas para a injeção da amostra, a lavagem do capilar e a troca de soluções para a análise eletroforética. Acima das peças mostradas nas Figuras $2 \mathrm{a}$ e 2b, encontram-se as guias para o capilar (b) confeccionadas em acrílico e borracha de silicone. As guias possibilitam uma maior estabilização da posição do capilar de sílica durante o movimento de elevação da peça na injeção da amostra. Com relação à automação do sistema, a interface PCI-1711 utilizada desempenha a função de aquisição do sinal do detector (voltagem) e de operação de todos os componentes do sistema via software no computador. As seguintes funções do sistema eletroforético são controladas pela placa da interface: fonte de alta tensão (controle da tensão de saída, monitoramento da tensão e corrente aplicadas, controle de polaridade e habilitação da saída da fonte); bomba de vácuo (liga/desliga); garra manipuladora do sistema de injeção (abre/fecha); altura e tempo de elevação do carro de impressora (driver do motor de passo); controlador de temperatura; ventiladores de circulação de ar (liga/desliga); amostradores (drivers dos motores de passo). O software para operação automatizada do sistema eletroforético através do computador foi desenvolvido em linguagem Delphi 7.0.
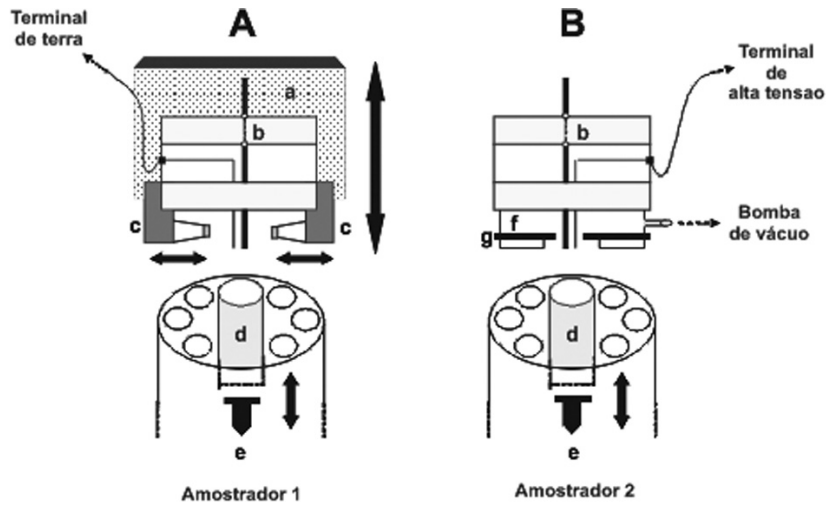

Figura 2. Representação esquemática das peças confeccionadas para o equipamento de eletroforese capilar para (A) injeção hidrodinâmica da amostra e (B) lavagem do capilar e aplicação de tensão: (a) carro de impressora com motor de passos, (b) guias de silicone para o capilar, (c) garra manipuladora, (d) reservatório da solução, (e) braço eletromecânico, $(f)$ bloco de acrílico para aplicação de vácuo, $(\mathrm{g})$ borracha sintética para vedação. As setas bilaterais representam os movimentos realizados por cada componente das peças projetadas

\section{Injeção da amostra}

Do lado esquerdo do equipamento (Figura 2A), pode-se ver o conjunto de peças projetadas para a injeção hidrodinâmica da amostra por elevação do reservatório contendo o capilar e o eletrodo de platina, bem como a garra manipuladora (c) que permite a fixação do reservatório (d) na exata altura onde se encontram o capilar e o eletrodo. O eletrodo de platina desta extremidade é conectado ao 
terminal de terra da fonte de alta tensão. O reservatório contendo a solução conecta-se a esta peça e encontra o capilar e o eletrodo pela elevação do braço eletromecânico (e) do carrossel de amostragem. O conjunto todo tanto pode ser mantido nesta posição, quando da análise eletroforética sob aplicação de tensão, como pode ser elevado até uma altura de $28 \mathrm{~cm}$ pelo carro da impressora (a), quando da injeção da amostra. A elevação da peça para a injeção da amostra é acionada por um motor de passos com driver de acionamento. Para o retorno à posição inicial, foi instalada uma chave de fim de curso no sistema de injeção. A altura e o tempo de injeção são programados por software e os pulsos de subida são dados por saída de pulsos da placa de interface. O sistema de injeção é controlado por uma placa modelo DL 100-CP (Rizzi \& Silveira Ltda., Santa Maria/RS, Brasil).

\section{Lavagem do capilar e aplicação da tensão}

Do lado direito do equipamento (Figura 2B), pode-se ver o conjunto de peças projetadas para a fixação do reservatório contendo o capilar e o eletrodo de platina, bem como o pequeno bloco de acrílico (f) vedado com borracha sintética (g) para a aplicação de vácuo. $\mathrm{O}$ reservatório contendo a solução (d) conecta-se a esta peça e encontra o capilar e o eletrodo pela elevação do braço eletromecânico (e) do carrossel. $\mathrm{O}$ eletrodo de platina desta extremidade é conectado ao terminal de tensão da fonte de alta tensão. A polaridade da fonte $(+$ ou -) é controlada através de saída digital. A habilitação da saída da fonte é ligada em série com um sensor magnético do tipo reed switch instalado na porta do equipamento. Este sensor só permite habilitar a fonte (fechamento do contato) com a porta do equipamento fechado, para evitar exposição do usuário aos altos potenciais empregados no equipamento. A bomba de vácuo é acionada apenas para a lavagem do capilar com água ou eletrólito de trabalho, empregando-se para isto uma pressão negativa de $-600 \mathrm{mmHg}$. $\mathrm{O}$ acionamento da bomba de vácuo é feito por uma contatora (LG modelo GMC-6M) comandada pela placa da interface (PCI-1711). A bomba de vácuo é ligada através de bornes no interior do quadro de comando do equipamento, sendo sua alimentação independente da alimentação do equipamento.

\section{Controle de temperatura}

Para o controle da temperatura do capilar e da solução no seu interior durante a aplicação da alta tensão de separação, o sistema dispõe de um sistema de aquecimento. Desta forma, o sistema de controle do equipamento acionará a resistência colocada na lateral do bloco de acrílico e o ventilador posicionado atrás da resistência fará circular ar quente pelo sistema, toda vez que a temperatura diminuir em $0,1^{\circ} \mathrm{C}$ a temperatura fixada no controlador. Os ventiladores posicionados na parte superior do bloco de acrílico auxiliam na circulação de ar e na estabilização da temperatura interna. $\mathrm{O}$ controlador de temperatura recebe o sinal do sensor de temperatura, indica a temperatura no painel (display), controla a temperatura através do acionamento da resistência de aquecimento e retransmite a temperatura para a placa de interface em sinal de 0 a $20 \mathrm{~mA}$. Na entrada analógica da placa de interface foi instalado um resistor shunt de $270 \Omega$ de precisão $1 \%$ para converter sinal de 0 a $20 \mathrm{~mA}$ em um sinal de 0 a 5,4 Vdc, compatível com a entrada analógica da placa. Desta forma, o sistema de controle estabiliza a temperatura interna do sistema eletroforético em relação à temperatura externa ao bloco de acrílico, a qual necessariamente deve ser sempre inferior à temperatura estabelecida do painel do controlador. A temperatura deve ser fixada em no mínimo $25^{\circ} \mathrm{C}$ para viabilizar o controle de temperatura em todas as estações do ano. Um sistema de controle de temperatura semelhante a este foi descrito na literatura por Böckel e colaboradores ${ }^{5}$ utilizando uma lâmpada e um ventilador para o aquecimento do sistema e a circulação de ar.

\section{Amostradores}

Os dois amostradores (Figuras 2A e 2B) construídos para o sistema, um para o lado da injeção de amostras e o outro para o lado da detecção, são constituídos basicamente de 2 partes: um carrossel contendo reservatórios e um braço eletromecânico para a elevação do reservatório e a introdução do capilar e do eletrodo de platina na solução. O carrossel é composto por um disco de acrílico, simetricamente perfurado em 9 posições, o qual é acoplado diretamente ao eixo de um motor de passos. A posição do carrossel é determinada por um sensor ótico (Photonic, modelo PHCT 09/99) localizado próximo à borda do disco de acrílico. Em cada posição perfurada há um pino de referência, sendo a posição zero marcada com 2 pinos para diferenciação dos demais. Para cada posição do carrossel foi construído um reservatório usinado em nylon para acomodação do reservatório da amostra (vial). Este reservatório de nylon (suporte do vial) tem um formato adequado para que o conjunto possa ser elevado e puxado de volta pelo mecanismo elevador, retornando à posição inicial. O mecanismo de elevação do reservatório foi construído usando-se um dispositivo eletromecânico de trava elétrica de porta de automóvel. O motor de corrente contínua que aciona este dispositivo foi substituído por um motor de passos. A extensão máxima do movimento do braço é de aproximadamente $3 \mathrm{~cm}$. Os drivers dos motores de passo dos dois amostradores são comandados por uma placa controladora DL 100-CP (Rizzi \& Silveira Ltda., Santa Maria, Brasil), com Software desenvolvido em linguagem C. A comunicação da placa controladora com o computador é feita via interface RS-232.

\section{Detecção condutométrica sem contato}

A construção do detector de condutividade está fundamentada no trabalho publicado por da Silva e do Lago, ${ }^{6}$ com alterações propostas por Böckel e colaboradores. ${ }^{7}$ A Figura 3 mostra de maneira esquemática os detalhes da célula de detecção. A detecção é composta dos seguintes estágios: entrada do sinal de alta frequência, eletrodo de aplicação do sinal de alta frequência, eletrodo de detecção, conversor corrente/tensão, retificador com amplificação de $10 \mathrm{x}$, filtro passa-baixas, buffer (ganho unitário), correção da linha de base com amplificação de 30x e circuito de proteção para a entrada da placa de interface (clamper). A placa foi instalada dentro de uma caixa metálica ligada ao potencial de terra para garantir a blindagem eletromagnética, minimizando a captação de ruído. Em torno do capilar foram construídos dois eletrodos cilíndricos usando fio de wrapping de 0,25 mm de diâmetro externo (código R30B-0100, OK Industries Inc. New York, USA). Um segmento de alguns centímetros deste fio foi enrolado, com o auxílio de ferramenta apropriada, em uma agulha descartável de $450 \mu \mathrm{m}$ que serve como molde, resultando em um tubo de alguns milímetros com diâmetro interno suficiente para passar o capilar com diâmetro externo de $360 \mu$ m. Os eletrodos foram soldados diretamente na placa de circuito impresso. Os eletrodos foram confeccionados com comprimento de $2 \mathrm{~mm}$ e soldados na placa com uma separação ( a ap de detecção) de $1 \mathrm{~mm}$. O sinal de onda senoidal de alta frequência proveniente do gerador de funções é aplicado a um dos eletrodos. O outro eletrodo é ligado ao conversor corrente/tensão e aos circuitos de amplificação e condicionamento do sinal (Figura 3). O sinal amplificado e condicionado ( $\mathrm{V}_{\mathrm{s}}$ ) é enviado ao software através da placa de interface conectada ao computador. Dois modelos de amplificadores operacionais adequados ao circuito foram utilizados: OPA2604AP (Texas instruments) e AD823AN (Analog Devices). Os resistores empregados no circuito são todos de precisão de $1 \%$. A alimentação do circuito é proveniente de uma fonte simétrica de $\pm 12 \mathrm{Vdc}$. 


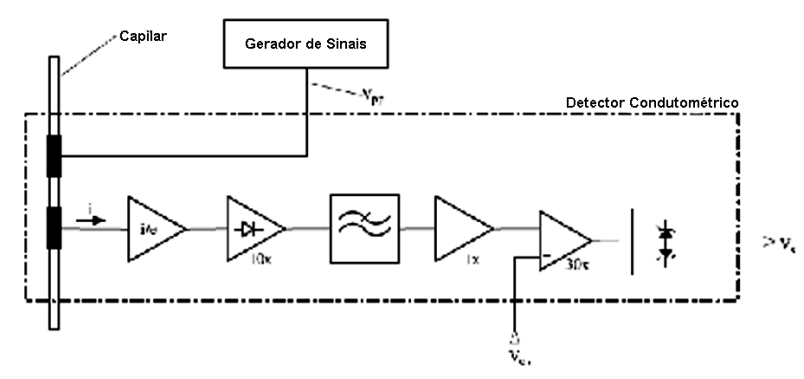

Figura 3. Representação esquemática do detector condutométrico construído para o equipamento de eletroforese capilar contendo capilar de sílica fundida, eletrodos e amplificadores operacionais

\section{PROCEDIMENTOS ANALÍTICOS}

\section{Reagentes e soluções}

Todas as soluções foram preparadas com água, destilada, deionizada e purificada (Milli-Q, Millipore, Bedford, USA), com resistividade de $18,2 \mathrm{M} \Omega \mathrm{cm}$. Todos os reagentes utilizados foram de grau analítico. As soluções estoque para análise dos cátions foram preparadas nas concentrações de $1 \mathrm{~g} \mathrm{~L}^{-1}$ a partir de $\mathrm{ZnSO}_{4} \cdot 7 \mathrm{H}_{2} \mathrm{O}$ (Merck), $\mathrm{NiCl}_{2} \cdot 6 \mathrm{H}_{2} \mathrm{O}$ (Merck), $\mathrm{CdSO}_{4} \cdot 8 \mathrm{H}_{2} \mathrm{O}$ (Merck), $\mathrm{Pb}\left(\mathrm{NO}_{3}\right)_{2}$ (Vetec), $\mathrm{Cr}\left(\mathrm{NO}_{3}\right)_{3} \cdot 9 \mathrm{H}_{2} \mathrm{O}$, (Vetec), $\mathrm{Co}\left(\mathrm{NO}_{3}\right)_{2} \cdot 6 \mathrm{H}_{2} \mathrm{O}$ (Nuclear), $\mathrm{TINO}_{3}$ (Aldrich) e $\mathrm{CuSO}_{4} \cdot 5 \mathrm{H}_{2} \mathrm{O}$ (Reagen). As amostras sintéticas dos cátions foram preparadas pela mistura e diluição das soluções estoque. As soluções do eletrólito de trabalho foram preparadas diariamente pela diluição das soluções estoque de $0,1 \mathrm{~mol} \mathrm{~L}^{-1}$ de ácido 2-N-morfolinoetanossulfônico (MES - Merck), histidina base livre (His - Sardi), ácido 2-hidroxi-isobutírico (HIBA - Sigma-Aldrich), ácido lático (HLac-Riedel-de Haën) e de éter 18-coroa-6 e metanol (MeOH), ambos da Merck. $\mathrm{O}$ valor do $\mathrm{pH}$ da solução do eletrólito de trabalho foi ajustado com $\mathrm{HNO}_{3}$ (Merck). As soluções foram armazenadas a $4{ }^{\circ} \mathrm{C}$ e ao abrigo da luz.

As soluções do eletrólito de trabalho foram filtradas usando membrana de acetato de celulose $0,45 \mu \mathrm{m}$ (Sartorius) em sistema sob vácuo e, em seguida, sonicadas por $30 \mathrm{~min}$.

\section{Análise de cátions metálicos no sistema $\mathrm{CE}-\mathrm{C}^{4} \mathrm{D}$}

O condicionamento do capilar de sílica fundida $(75 \mu \mathrm{m}$ de diâmetro interno, $360 \mu \mathrm{m}$ de diâmetro externo e $68 \mathrm{~cm}$ de comprimento) foi feito em fluxo aplicando-se uma pressão de $-600 \mathrm{mmHg}$ na extremidade próxima ao detector. Primeiramente, empregou-se $\mathrm{NaOH}$ (Merck), na concentração $1 \mathrm{~mol} \mathrm{~L}^{-1}$, durante 40 min, seguido de água purificada por $10 \mathrm{~min}, \mathrm{NaOH} 0,1 \mathrm{~mol} \mathrm{~L}^{-1}$ por $15 \mathrm{~min}$, água purificada por 10 min e, finalmente, solução do eletrólito de trabalho por $30 \mathrm{~min}$. O potencial de $15 \mathrm{kV}$ foi aplicado até a estabilização da linha base. Entre as injeções das amostras, o capilar foi lavado com o eletrólito de trabalho durante 5 min mediante aplicação de vácuo, nas mesmas condições descritas acima. Ao final de um dia de trabalho, o capilar era lavado com água ultrapura por $15 \mathrm{~min}$.

\section{RESULTADOS E DISCUSSÃO}

\section{Separação simultânea de $\mathrm{Na}^{+}, \mathrm{K}^{+}, \mathrm{Ca}^{2+}$ e $\mathrm{Mg}^{2+}$ por $\mathrm{CE}-\mathrm{C}^{4} \mathrm{D}$}

Após a sua montagem e configuração, o sistema CE-C ${ }^{4} \mathrm{D}$ foi testado de acordo com metodologias previamente descritas na literatura para a separação de metais alcalinos e alcalinoterrosos. ${ }^{5,7,9}$ A separação eletroforética dos metais $\mathrm{Na}^{+}, \mathrm{K}^{+}, \mathrm{Ca}^{2+}$ e $\mathrm{Mg}^{2+}$ foi feita pela aplicação de um potencial de $15 \mathrm{kV}$, com o detector $\mathrm{C}^{4} \mathrm{D}$ operando em $600 \mathrm{kHz}$ e $2 \mathrm{~V}_{\mathrm{pp}}$ e utilizando-se uma solução tampão MES/His $0,02 \mathrm{~mol} \mathrm{~L}^{-1}$ (pH 5,0) como eletrólito de trabalho. A repetibilidade do sistema foi avaliada pela injeção sequencial (injeção hidrodinâmica por gravidade a $10 \mathrm{~cm}$ por 30 s) de uma solução aquosa contendo uma mistura de $\mathrm{Na}^{+}, \mathrm{K}^{+}, \mathrm{Ca}^{2+}$ e $\mathrm{Mg}^{2+}$ nas seguintes concentrações: $100 \mu \mathrm{mol} \mathrm{L}{ }^{-1}$ para $\mathrm{K}^{+} \mathrm{e} \mathrm{Ca}^{2+}$ e $170 \mu \mathrm{mol} \mathrm{L} \mathrm{L}^{-1}$ para $\mathrm{Na}^{+}$e $\mathrm{Mg}^{2+}$. Os tempos de migração (tm) para os quatro picos foram medidos para cinco injeções sucessivas e seus respectivos valores de desvio padrão estão listados na Tabela 1 . Os coeficientes de variação calculados apresentam valores entre 6,73 e 9,19\% para os tm. Estes resultados estão de acordo os encontrados na literatura; $;^{5,79}$ ou seja, há uma boa resolução e reprodutibilidade dos picos para os quatro cátions, indicando o desempenho adequado do sistema CE-C ${ }^{4} \mathrm{D}$ construído em laboratório. Nesta tabela também estão descritos os valores de limites de detecção (LOD) e de quantificação (LOQ) calculados para os quatro cátions citados.

Tabela 1. Valores de tempos de migração, limites de detecção e quantificação para os cátions $\mathrm{K}^{+}, \mathrm{Ca}^{2+}, \mathrm{Na}^{+}$e $\mathrm{Mg}^{2+}(n=5)$; Eletrólito de trabalho: MES/His $20 \mathrm{mmol} \mathrm{L}^{-1}$ (pH 5,0); Potencial de separação: $-15 \mathrm{kV}$; Temperatura: $25^{\circ} \mathrm{C}$; Injeção hidrodinâmica (gravidade): 10 cm por $30 \mathrm{~s}$; Capilar: sílica fundida de $68 \mathrm{~cm}$ com $46 \mathrm{~cm}$ até o detector (75 $\mu \mathrm{m}$ diâmetro interno x $360 \mu \mathrm{m}$ diâmetro externo)

\begin{tabular}{|c|c|c|c|c|}
\hline Parâmetros & $\mathrm{K}^{+}$ & $\mathrm{Ca}^{2+}$ & $\mathrm{Na}^{+}$ & $\mathrm{Mg}^{2+}$ \\
\hline Tempo de migração (min) & 3,71 & 4,22 & 4,35 & 4,55 \\
\hline Desvio padrão (min) & $\pm 0,25$ & $\pm 0,30$ & $\pm 0,40$ & $\pm 0,29$ \\
\hline $\mathrm{LOD}\left(\mu \mathrm{mol} \mathrm{L} \mathrm{L}^{-1}\right)$ & 1,90 & 1,54 & 1,84 & 1,36 \\
\hline $\mathrm{LOQ}\left(\mu \mathrm{mol} \mathrm{L}{ }^{-1}\right)$ & 5,70 & 4,62 & 5,49 & 4,08 \\
\hline
\end{tabular}

Separação simultânea de $\mathrm{Tl}^{+}, \mathrm{Pb}^{2+}, \mathrm{Cd}^{2+}, \mathrm{Cr}^{3+}, \mathrm{Zn}^{2+}, \mathrm{Co}^{2+}, \mathrm{Cu}^{2+} \mathrm{e}$ $\mathrm{Ni}^{2+}$ por $\mathrm{CE}-\mathrm{C}^{4} \mathrm{D}$

O sistema CE- $C^{4} \mathrm{D}$ construído foi empregado também para a separação de cátions metálicos do grupo dos metais de transição. Devido às semelhanças nos valores de mobilidade eletroforética para alguns cátions deste grupo selecionado, a separação eletroforética envolve normalmente o uso de aditivos no eletrólito de trabalho, como complexantes e solventes orgânicos. Portanto, uma etapa subsequente do trabalho envolveu a aplicação do sistema construído na otimização da separação destes cátions por CZE.

Sabendo-se que o pH do eletrólito de trabalho é o parâmetro de separação mais importante em CE para influenciar na seletividade do sistema, a separação simultânea dos cátions $\mathrm{Tl}^{+}, \mathrm{Pb}^{2+}, \mathrm{Cd}^{2+}, \mathrm{Cr}^{3+}$, $\mathrm{Zn}^{2+}, \mathrm{Co}^{2+}, \mathrm{Cu}^{2+}$ e $\mathrm{Ni}^{2+}$ foi investigada em diferentes valores de $\mathrm{pH}$ do tampão MES/His $0,02 \mathrm{~mol} \mathrm{~L}^{-1}$. Os resultados obtidos para os oito cátions em valores de $\mathrm{pH}$ entre 5,0 e 6,1 mostraram que a variação deste modifica significativamente os tempos de migração (tm) obtidos. Por exemplo, em valores de $\mathrm{pH}$ 5,0 e 5,5 o íon $\mathrm{Ni}^{2+}$ foi o último pico visualizado no eletroferograma, bem diferente do resultado encontrado em pH 6,1 no qual sua zona passa a sair junto com o plug da água e, desta forma, o seu pico não foi detectado. Isto ocorreu pelo fato de que em um valor de $\mathrm{pH}$ maior a concentração de íons $\mathrm{Ni}^{2+}$ livres é menor, já que nestas condições existe uma concentração maior desses íons na forma de hidróxido. Apesar da investigação do pH do eletrólito de trabalho nesta faixa descrita não fornecer uma separação completa dos oito cátions estudados, o eletrólito de trabalho MES/ His $0,02 \mathrm{~mol} \mathrm{~L}^{-1} \mathrm{em} \mathrm{pH} \mathrm{5,0} \mathrm{foi} \mathrm{escolhido} \mathrm{para} \mathrm{dar} \mathrm{continuidade} \mathrm{aos}$ estudos de otimização, pelo fato de fornecer um tempo de análise em torno de $10 \mathrm{~min}$. 
Além do pH, a força iônica do eletrólito é um parâmetro importante que pode ser variado para melhorar a eficiência de separação, a resolução dos picos e a sensibilidade do sistema de detecção. A dependência dos tempos de migração dos cátions $\mathrm{Tl}^{+}, \mathrm{Pb}^{2+}, \mathrm{Cd}^{2+}$, $\mathrm{Cr}^{3+}, \mathrm{Zn}^{2+}, \mathrm{Co}^{2+}, \mathrm{Cu}^{2+}$ e Ni ${ }^{2}$ com a concentração de MES/His em $\mathrm{pH}$ 5,0 foi investigada na faixa de concentração de 0,01 a $0,05 \mathrm{~mol} \mathrm{~L}^{-1}$. Aqui, observou-se que o aumento da concentração do eletrólito de trabalho de 0,01 para $0,03 \mathrm{~mol} \mathrm{~L}^{-1}$ leva a uma melhora significativa na separação dos picos dos oito cátions, apesar do tempo de análise ser maior. Por outro lado, a altura dos picos diminuiu com o aumento da força iônica do eletrólito, devido à diminuição da relação sinal/ ruído pelo aumento da condutividade basal. Portanto, os testes subsequentes de otimização da separação dos cátions foram realizados comparativamente para eletrólitos de MES/His nas concentrações 0,02 e $0,03 \mathrm{~mol} \mathrm{~L}^{-1}$ (ambos em pH 5,0).

A sensibilidade da $\mathrm{C}^{4} \mathrm{D}$ em CZE está diretamente ligada à diferença de condutividade entre o eletrólito de trabalho e a zona do soluto que migra pelo capilar. Portanto, a separação dos cátions foi investigada também em eletrólitos de trabalho de diferentes composições. Para avaliar a capacidade de separação dos eletrólitos testados, uma amostra padrão contendo $\mathrm{Tl}^{+}, \mathrm{Pb}^{2+}, \mathrm{Cd}^{2+}, \mathrm{Cr}^{3+}, \mathrm{Zn}^{2+}, \mathrm{Co}^{2+}, \mathrm{Cu}^{2+} \mathrm{e} \mathrm{Ni}^{2+}$ na concentração $50 \mu \mathrm{mol} \mathrm{L}^{-1}$ (cada) foi analisada. Primeiramente, metanol (1 a $20 \% \mathrm{v} / \mathrm{v}$ ) foi adicionado aos eletrólitos MES/His nas concentrações de 0,02 e $0,03 \mathrm{~mol} \mathrm{~L}^{-1}$ (pH 5,0). Comparando-se os eletroferogramas obtidos com estes eletrólitos, uma separação eficiente dos oito cátions foi obtida com MES/His $0,02 \mathrm{~mol} \mathrm{~L}^{-1}$ ( $\left.\mathrm{pH} 5,0\right)$ contendo MeOH $5 \%$ (v/v) como aditivo. O solvente orgânico promoveu a modificação da viscosidade da solução do eletrólito de trabalho, o que ocasionou mudanças de mobilidade aparente e, portanto, na migração relativa dos cátions. A adição de agentes complexantes ao eletrólito de trabalho também foi investigada, onde se observou que o complexante ácido 2-hidroxiisobutírico (HIBA) promoveu o aumento nos tempos de migração de todos os cátions, exceto o $\mathrm{Tl}^{+}$. Entretanto, a sensibilidade na detecção (relação sinal/ruído) obtida com o eletrólito de trabalho MES/His 0,02 mol L ${ }^{-1}\left(\mathrm{pH} \mathrm{5,0)} \mathrm{contendo} \mathrm{HIBA} 1 \mathrm{mmol} \mathrm{L}^{-1}\right.$ foi inferior àquela obtida com o eletrólito MES/His $0,02 \mathrm{~mol} \mathrm{~L}^{-1}$ (pH 5,0) contendo $\mathrm{MeOH} 5 \%$ $(\mathrm{v} / \mathrm{v})$ como aditivo. Outros testes envolveram a troca do ácido orgânico do eletrólito, MES, por ácido lático (HLac) e HIBA. As melhores condições para a separação com estes eletrólitos foram obtidas para o eletrólito HLac/His $0,02 \mathrm{~mol} \mathrm{~L}^{-1}$ (pH 5,25) contendo $\mathrm{MeOH} 10 \%$ (v/v), onde significativas modificações no comportamento eletroforético dos cátions foram observadas. Aqui, picos negativos foram registrados para $\mathrm{Zn}^{2+} \mathrm{e} \mathrm{Co}^{2+}$, pois suas zonas passaram a ter condutividades menores que a do eletrólito de trabalho, além do cátion $\mathrm{Co}^{2+}$ comigrar com o cátion $\mathrm{Ni}^{2+}$. A separação com o eletrólito composto por HIBA/His 0,01 $\mathrm{mol} \mathrm{L}^{-1}(\mathrm{pH} 4,85)$ e $\mathrm{MeOH} 10 \%$ (v/v) foi a menos eficiente de todas as investigadas devido à baixa resolução entre os picos obtidos para os pares $\mathrm{Zn}^{2+} / \mathrm{Co}^{2+} \mathrm{e} \mathrm{Ni}^{2+} / \mathrm{Cu}^{2+}$, além das comigrações observadas para $\mathrm{Cd}^{2+} \mathrm{e} \mathrm{Cr}^{3+}$. A adição de éter 18-coroa-6 como agente complexante ao eletrólito de trabalho MES/His $0,02 \mathrm{~mol} \mathrm{~L}^{-1}$ (pH 5,0) e MeOH $5 \%$ (v/v) também foi investigada. Entretanto, esta condição originou picos com alturas menores em relação aos outros eletrólitos investigados, além de apresentar baixa resolução entre os picos detectados. Pela comparação dos eletroferogramas obtidos para as misturas sintéticas dos cátions, observou-se que o eletrólito de trabalho contendo MES/His 0,02 mol $\mathrm{L}^{-1}(\mathrm{pH} 5,0)$ e $\mathrm{MeOH} 5 \%(\mathrm{v} / \mathrm{v})$ forneceu a melhor condição para a separação simultânea de $\mathrm{Tl}^{+}, \mathrm{Pb}^{2+}, \mathrm{Cd}^{2+}, \mathrm{Cr}^{3+}, \mathrm{Zn}^{2+}, \mathrm{Co}^{2+}, \mathrm{Cu}^{2+}$ e $\mathrm{Ni}^{2+}$ utilizando-se um capilar de sílica fundida de $68 \mathrm{~cm}$ de comprimento ( $46 \mathrm{~cm}$ até o detector) e $75 \mu \mathrm{m}$ de diâmetro interno, com boa resolução de picos e maior relação sinal/ruído para todos os cátions estudados. A separação eletroforética dos oito cátions sob condições otimizadas pode ser vista nas Figuras 4 e 5 em uma amostra sintética e uma amostra real de água de hemodiálise contaminada com os cátions. Como pode ser observado, a determinação destas espécies é possível na presença de íons $\mathrm{Na}^{+}$presentes na água de hemodiálise.

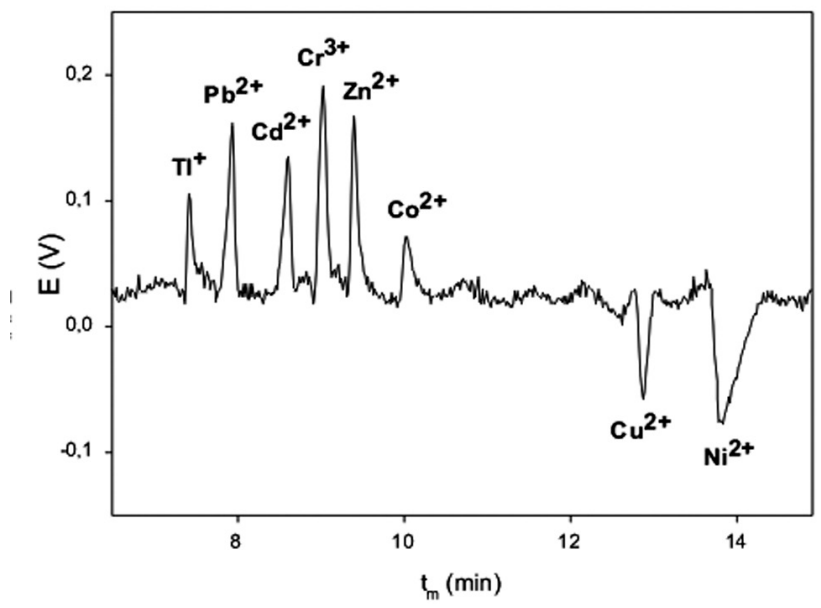

Figura 4. Eletroferograma de uma amostra sintética de $4 \mathrm{mg} \mathrm{L}^{-1}$ de Tl(I), $\mathrm{Pb}(\mathrm{II}), \mathrm{Cd}(\mathrm{II}), \mathrm{Cr}(\mathrm{III}), \mathrm{Zn}(\mathrm{II}), \mathrm{Co}(\mathrm{II}), \mathrm{Cu}(\mathrm{II})$ e Ni(II) em eletrólito de trabalho MES/His 0,02 mol L $L^{-1}$ (pH 5,0) e MeOH 5\% (v/v), com aplicação de $10 \mathrm{kV}$ para a separação dos metais. Capilar: sílica fundida $(75 \times 360 \mu \mathrm{m})$ de 68 $\mathrm{cm}$ de comprimento com $46 \mathrm{~cm}$ até o detector; Detector: $C^{4} D$ operando em $600 \mathrm{kHz}$ e $2 \mathrm{~V}_{p p}$; Temperatura de separação: $25^{\circ} \mathrm{C}$; Injeção hidrodinâmica por gravidade: $28 \mathrm{~cm}$ por $60 \mathrm{~s}$

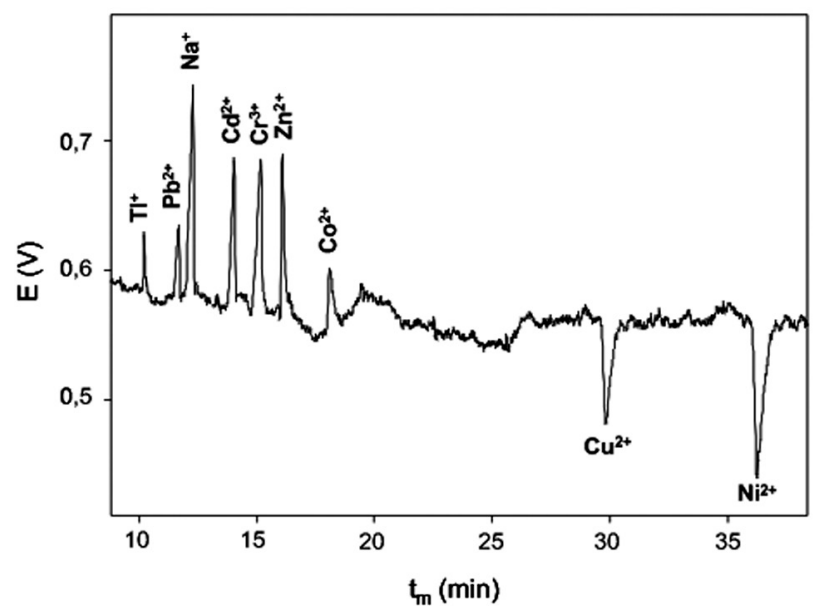

Figura 5. Eletroferograma de uma amostra de água de diálise contaminada com $4 \mathrm{mg} \mathrm{L}^{-1}$ de Tl(I), Pb(II), Cd(II), Cr(III), Zn(II), Co(II), Cu(II) e Ni(II) em eletrólito de trabalho de MES/His 0,02 $\mathrm{mol} \mathrm{L}^{-1}$ (pH 5,0) e MeOH 10\% (v/v), com aplicação de $10 \mathrm{kV}$ para a separação dos metais. Capilar: sílica fundida, 75 um, $68 \mathrm{~cm}, 46 \mathrm{~cm}$ até o detector; Detector: $C^{4} D$ operando em $600 \mathrm{kHz}$ e $2 \mathrm{~V}_{p p}$; Temperatura: $25^{\circ} \mathrm{C}$; Injeção hidrodinâmica por gravidade: $28 \mathrm{~cm}$ por $60 \mathrm{~s}$

A repetibilidade do sistema foi avaliada pela injeção sequencial (injeção hidrodinâmica por gravidade a $28 \mathrm{~cm}$ por $60 \mathrm{~s}$ ) de uma solução aquosa contendo uma mistura de $\mathrm{Tl}^{+}, \mathrm{Pb}^{2+}, \mathrm{Cd}^{2+}, \mathrm{Cr}^{3+}, \mathrm{Zn}^{2+}$, $\mathrm{Co}^{2+}, \mathrm{Cu}^{2+} \mathrm{e} \mathrm{Ni}^{2+}$ na concentração $4 \mathrm{mg} \mathrm{L}^{-1}$. Os tempos de migração para os oito cátions foram medidos para cinco injeções sucessivas e seus respectivos valores de desvio padrão estão listados na Tabela 2. Os coeficientes de variação calculados apresentam valores médios de 2,8\% para os tempos de migração dos metais. O limite de detecção (LOD) para os cátions foi calculado a partir equação da curva analítica $\left(3 \sigma_{\mathrm{B}} / \mathrm{b}\right)$ após 10 medidas do branco. Os resultados podem ser visualizados na Tabela 2 . O valor da área dos picos obtidos na 
Tabela 2. Figuras de mérito para a determinação simultânea ( $n=5)$ dos cátions $\mathrm{Tl}^{+}, \mathrm{Pb}^{2+}, \mathrm{Cd}^{2+}, \mathrm{Cr}^{3+}, \mathrm{Zn}^{2+}, \mathrm{Co}^{2+}, \mathrm{Cu}^{2+}, \mathrm{Ni}^{2+}$ por $\mathrm{CZE}^{2} \mathrm{CCD}$. Eletrólito de trabalho: MES/His $20 \mathrm{mmol} \mathrm{L}{ }^{-1}\left(\mathrm{pH} \mathrm{5,0)} \mathrm{e} \mathrm{MeOH} \mathrm{5 \%} \mathrm{(v/v);} \mathrm{Potencial} \mathrm{de} \mathrm{separação:}-10 \mathrm{kV}\right.$; Temperatura: $25{ }^{\circ} \mathrm{C}$; Injeção hidrodinâmica (gravidade): $28 \mathrm{~cm}$ por $60 \mathrm{~s}$; Capilar: sílica fundida de $68 \mathrm{~cm}$ com $46 \mathrm{~cm}$ até o detector $(75 \mu \mathrm{m}$ diâmetro interno x $360 \mu \mathrm{m}$ diâmetro externo)

\begin{tabular}{lcccccccc}
\hline Parâmetros & $\mathrm{Tl}^{+}$ & $\mathrm{Pb}^{2+}$ & $\mathrm{Cd}^{2+}$ & $\mathrm{Cr}^{3+}$ & $\mathrm{Zn}^{2+}$ & $\mathrm{Co}^{2+}$ & $\mathrm{Cu}^{2+}$ & $\mathrm{Ni}^{2+}$ \\
\hline Tempo de migração (min) ${ }^{a}$ & 7,64 & 7,83 & 8,68 & 9,12 & 9,43 & 10,09 & 12,93 & 13,77 \\
Desvio padrão relativo (\%) & 2,7 & 2,9 & 2,8 & 2,7 & 3,0 & 2,6 & 2,9 & 3,4 \\
Área média (V min) $^{a}$ & 0,0042 & 0,0049 & 0,014 & 0,0200 & 0,0114 & 0,00130 & 0,0162 & 0,0305 \\
Desvio padrão relativo (\%) & 2,3 & 2,0 & 2,1 & 4,5 & 4,0 & 4,1 & 2,4 & 2,9 \\
LOD $\left(\mu \mathrm{mol} \mathrm{L}^{-1}\right.$ ) & 5,08 & 1,69 & 3,52 & 5,93 & 5,16 & 22,17 & 8,43 & 3,28 \\
LOQ $\left(\mu \mathrm{mol} \mathrm{L}^{-1}\right)$ & 16,91 & 5,62 & 11,72 & 19,74 & 17,02 & 73,82 & 28,07 & 10,92 \\
\hline
\end{tabular}

${ }^{a} n=5$

detecção condutométrica sem contato forneceu uma resposta linear para todos os cátions nas seguintes faixas de concentração $(n=5)$ : 17-280 $\mu \mathrm{mol} \mathrm{L}^{-1}$ para $\mathrm{Tl}^{+}$e $\mathrm{Zn}^{2+} ; 12-280 \mu \mathrm{mol} \mathrm{L}^{-1}$ para Cd $\mathrm{Cd}^{2+} ; 74-300$ $\mu \mathrm{mol} \mathrm{L}{ }^{-1}$ para $\mathrm{Co}^{2+} ; 6-300 \mu \mathrm{mol} \mathrm{L}-1$ para $\mathrm{Pb}^{2+} ; 20-350 \mu \mathrm{mol} \mathrm{L}{ }^{-1}$ para $\mathrm{Cr}^{3+}$; 28-320 $\mu \mathrm{mol} \mathrm{L} \mathrm{L}^{-1}$ para $\mathrm{Cu}^{2+}$ e $11-350 \mu \mathrm{mol} \mathrm{L}{ }^{-1}$ para $\mathrm{Ni}^{2+}$.

\section{CONCLUSÕES}

O sistema CE-C ${ }^{4} \mathrm{D}$ descrito neste trabalho emprega um sistema de manipulação de soluções completamente automatizado empregando dois carrosséis de amostras acoplados ao equipamento. Todos os componentes do sistema eletroforético são operados através de uma interface via computador, incluindo os dois amostradores projetados e acoplados ao sistema eletroforético. O detector condutométrico foi construído de acordo com protótipos previamente descritos na literatura e permite a detecção sensível de metais alcalinos, alcalino-terrosos e metais de transição. O sistema construído em laboratório apresenta boa reprodutibilidade para os tempos de migração dos metais estudados, com desvio padrão relativo (RSD) entre 6,3 e 9,2\% para $\mathrm{Na}^{+}, \mathrm{K}^{+}, \mathrm{Ca}^{2+}$ e $\mathrm{Mg}^{2+}$ e entre 2,60 e 3,4\% para $\mathrm{Zn}^{2+}, \mathrm{Cr}^{3+}, \mathrm{Pb}^{2+}, \mathrm{Cd}^{2+}, \mathrm{Co}^{2+}, \mathrm{Cu}^{2+}, \mathrm{Ni}^{2+} \mathrm{e} \mathrm{Tl}^{+}$. O método eletroforético desenvolvido foi empregado na otimização da separação dos cátions metálicos $\mathrm{Zn}^{2+}, \mathrm{Cr}^{3+}, \mathrm{Pb}^{2+}, \mathrm{Cd}^{2+}, \mathrm{Co}^{2+}, \mathrm{Cu}^{2+}, \mathrm{Ni}^{2+}$ e $\mathrm{Tl}^{+}$em amostras de água. O eletrólito de trabalho composto pelo tampão MES/His 0,02 mol $\mathrm{L}^{-1}$ (pH 5,0) e MeOH 5\% (v/v) possibilitou a separação simultânea dos cátions metálicos empregando um capilar de sílica fundida de $68 \mathrm{~cm}$ com $46 \mathrm{~cm}$ até o detector (75 بm diâmetro interno x $360 \mu \mathrm{m}$ diâmetro externo) e um potencial de separação de $-10 \mathrm{kV}$. Limites de detecção calculados para os oitos cátions metálicos entre 1,69 e 22,17 $\mu \mathrm{mol} \mathrm{L^{-1 }}$ permitem a aplicação da metodologia para determinações em amostras reais empregando o sistema eletroforético desenvolvido.

\section{AGRADECIMENTOS}

À FAPERGS (PROAPP processo 0410870), CNPq (processo 478215/2007-0), CAPES (PROBRAL 204/06) pelos auxílios e bol- sas concedidas. Agradecimentos especiais são feitos ao Engenheiro eletricista L. Schirmer pelo suporte técnico, à Dra. C. Piatnicki e ao Ms. W. Böckel do IQ-UFRGS pela valiosa troca de idéias na construção do equipamento.

\section{REFERÊNCIAS}

1. Jager, A. V.; Tavares, M. F. M.; Quim. Nova 2001, 24, 363.

2. Tavares, M. F. M.; Quim. Nova 1997, 20, 493.

3. Hjertén, S.; Chromatogr. Rev. 1967, 9, 122.

4. Da Silva, J. A. F.; Tese de Doutorado, Universidade de São Paulo, Brasil, 2001.

5. Da Silva, J. A. F.; Do Lago, C. L.; Anal. Chem. 1998, 70, 4339.

6. Da Silva, J. A. F.; Guzman, N.; Do Lago, C. L.; J. Chromatogr., A 2002, 942, 249.

7. Böckel, W. J.; Martini, E. M. A.; Santos, D.; Piatnicki, C. M. S.; Quim. Nova 2005, 28, 1106

8. Gas, B.; Demjanenko, M.; Vacík, J.; J. Chromatogr., A 1980, 192, 253.

9. Zemann, A. J.; Schnell, E.; Volgger, D.; Bonn, G. K.; Anal. Chem. 1998, $70,563$.

10. Tanyanyiwa, J.; Hauser, P. C.; Anal. Chem. 2002, 74, 6378.

11. Johnston, S. E.; Fadgen, K. E; Tolley, L. T.; Jorgenson, J. W.; J. Chromatogr., A 2005, 1094, 148.

12. Tan, F.; Yan, B.; Guan, Y.; Anal. Sci. 2005, 21, 955.

13. Kuban, P.; Karlberg, B.; Kuban, P.; Kuban, V.; J. Chromatogr., A 2002, 964, 227

14. Da Silva, J. A. F.; Ricelli, N. L.; Carvalho, A. Z.; Do Lago, C. L.; J. Braz. Chem. Soc. 2003, 14, 265.

15. Mayrhofer, K.; Zemann, A. J.; Schnell, E.; Bonn, G. K.; Anal. Chem. 1999, 71, 3828.

16. Tavares, M. F. M.; Quim. Nova 1996, 19, 173.

17. Da Silva, J. A. F.; Quim. Nova 2003, 26, 56.

18. Da Silva, J. A. F.; Coltro, W. K. T.; Carrilho, E.; Tavares, M. F. M.; Quim. Nova 2007, 30, 744 . 